\title{
Enhancement in physical properties of barium hexaferrite with substitution - ERRATUM
}

Talwinder Kaur, Sachin Kumar, Bilal H. Bhat, and Ajeet Kumar Srivastava

doi: 10.1557/jmr.2015.244, Published by Materials Research Society with Cambridge University Press, 20 August 2015.

In Kaur ${ }^{1}$, the author Ajeet Kumar Srivastava's name was listed as Ajeet Kumar Srivastava Srivastava.

The original has since been corrected.

\section{REFERENCE}

1. Talwinder Kuar, Sachin Kumar, Bilal H. Bhat, and Ajeet Kumar Srivastava: Enhancement in physical properties of barium hexaferrite with substitution. J. Mater. Res. 30(18) (2015). 